IPM/P-2009/008

\title{
On AdS/CFT of Galilean Conformal Field Theories
}

\author{
Mohsen Alishahiha ${ }^{a}$, Ali Davody ${ }^{a, b}$ and Ali Vahedi ${ }^{a, b}$ \\ a School of physics, Institute for Research in Fundamental Sciences (IPM) \\ P.O. Box 19395-5531, Tehran, Iran \\ ${ }^{b}$ Department of Physics, Sharif University of Technology \\ P.O. Box 11365-9161, Tehran, Iran \\ alishah, davody, vahedi@ipm.ir
}

\begin{abstract}
We study a new contraction of a $d+1$ dimensional relativistic conformal algebra where $n+1$ directions remain unchanged. For $n=0,1$ the resultant algebras admit infinite dimensional extension containing one and two copies of Virasoro algebra, respectively. For $n>1$ the obtained algebra is finite dimensional containing an $s o(2, n+1)$ subalgebra. The gravity dual is provided by taking a Newton-Cartan like limit of the Einstein's equations of AdS space which singles out an $A d S_{n+2}$ spacetime. The infinite dimensional extension of $n=0,1$ cases may be understood from the fact that the dual gravities contain $A d S_{2}$ and $A d S_{3}$ factor, respectively. We also explore how the AdS/CFT correspondence works for this case where we see that the main role is playing by $A d S_{n+2}$ base geometry.
\end{abstract}




\section{Introduction}

Non-relativistic AdS/CFT correspondence has recently been studied in several papers including [1] - [37]. Actually non-relativistic CFTs may be obtained from relativistic CFTs by making use of a non-relativistic limit. In general by taking a non-relativistic limit it means that we are sending the speed of light to infinity. More precisely we have $v / c \rightarrow 0$ where $v$ is the typical speed of the model. We note, however, that there are several ways to take this limit which may even reduce the dimensions of the spacetime too.

To explore the procedures of taking the non-relativistic limit we will start from a relativistic CFT in $d+1$ dimensions parametrized by $t, x_{i}$ for $i=1, \cdots, d$. To proceed let us decompose the coordinates as $\left(x^{+}, x^{-}, x_{i}\right), i=1, \cdots, d-1$, where the light like coordinates $\left(x^{+}, x^{-}\right)$are defined by

$$
x^{+}=\frac{1}{\sqrt{2}}\left(t+x_{d}\right), \quad x^{-}=\frac{1}{\sqrt{2}}\left(t-x_{d}\right) .
$$

Next we compactify the light like coordinate $x^{-}$and identify the momentum along the light like coordinate with the number operator of the non-relativistic CFT. Then we look for those generators of the relativistic conformal algebra that commute with the number operator which altogether construct an algebra. The resultant algebra is the Schrödinger algebra $[38,39]$ which is the symmetry of the Schrödinger equation. In other words the Schrödinger group may be thought of as a subgroup of $S O(2, d+1)$ with fixed momentum along the null direction (see for example [40-45]). A theory with this symmetry is a nonrelativistic CFT with the following scaling symmetry

$$
x^{+} \rightarrow \lambda^{2} x^{+}, \quad x_{i} \rightarrow \lambda x_{i} .
$$

Note that starting from $d+1$ dimensional relativistic CFT the obtained theory is a nonrelativistic CFT in $d$ dimensions. This symmetry, for example, is relevant to study cold atoms [1]. The generators of the corresponding algebra are spatial translations $P_{i}$, rotations $M_{i j}$, time translation $H$, Galilean boosts $B_{i}$, dilation $D$, number operator $N$ and special conformal transformation $K$. The algebra has also a central extension given by the number operator.

Recently gravity duals of non-relativistic CFTs have been proposed in [1,2]. It has also been shown [46] that the asymptotic symmetry algebra of the corresponding geometry, in any dimension, is an infinite dimensional algebra containing one copy Virasoro algebra compatible with the symmetry of non-relativistic CFT [47].

On the other hand one may look for a non-relativistic conformal algebra which scales space and time in the same way

$$
t \rightarrow \lambda t, \quad x_{i} \rightarrow \lambda x_{i}
$$

This algebra has recently been studied in [33](see also [48]) where it was shown that the corresponding algebra may be obtained from $d+1$ dimensional relativistic conformal algebra by making use of a contraction. Since the contraction does not change the dimension of the algebra the resultant algebra can be thought of as the symmetry of a non-relativistic CFT in $d+1$ dimensions. More precisely the contraction can be defined by the scaling 
$t \rightarrow t, x_{i} \rightarrow \epsilon x_{i}$ in the limit of $\epsilon \rightarrow 0$. The generators of the obtained algebra are spatial translations, $P_{i}$, rotations $J_{i j}$, time translation $H$, Galilean boosts $B_{i}$, dilation $D$, special conformal transformation $K$ and spatial special conformal transformation $K_{i}$.

It is also shown [33] that the corresponding algebra admits an infinite dimensional extension containing one copy of Virasoro algebra and the bulk gravity dual is provided by a Newtonian gravity given in terms of a non-dynamical metric but a dynamical torsion free affine connections. The gravity background may also be thought of as spatial $d-1$ dimensional space fibered over an $A d S_{2}$. In this sense the infinite dimensional extension may be understood from the asymptotic isometries of this $A d S_{2}$.

The aim of this article is to extend the above considerations for a new contraction in which we scale $d-n-1$ directions by $\epsilon$ and $n+1$ directions remain unchanged. Then we consider the limit of $\epsilon \rightarrow 0$. The obtained algebra which we call it semi-Galilean algebra can be thought of as a symmetry of non-relativistic CFT in $d+1$ dimensions 1 .

We show that for $n=1$ the corresponding algebra admits an infinite dimensional extension containing two copies of Virasoro algebra. The corresponding gravity dual is defined on a geometry which is a $d-2$ dimensional spatial space fibered over an $A d S_{3}$ and, indeed, the infinite dimensional extension can be associated to the asymptotic isometries of $A d S_{3}$.

For $n \geq 2$ the contraction leads to an algebra which has $s o(2, n+1) \times \operatorname{so}(d-n-1)$ subalgebra and the corresponding gravity dual is defined by a geometry which is a $d-n-1$ dimensional spatial space fibered over an $A d S_{n+2}$. The subalgebra can, then, be identified with the isometries of $A d S_{n+2}$. Since the asymptotic symmetry algebra of $A d S_{n+2}$ space for $n \geq 2$ is finite dimensional, the corresponding semi-Galilean algebra is also finite dimensional.

The paper is organized as follows. In the next section we study the Galilean algebra for arbitrary $n$. In section three we explore how the AdS/CFT correspondence works in this context. The last section is devoted to discussions.

\section{General contraction of conformal algebra}

In this section we study non-relativistic limit of relativistic conformal algebra in $d+1$ dimensions by making use of a contraction. To proceed we consider the following general scaling

$$
t \rightarrow t, \quad y_{\alpha} \rightarrow y_{\alpha}, \quad x_{i} \rightarrow \epsilon x_{i},
$$

where $\alpha=1, \cdots, n$ and $i=n+1, \cdots d$. The contraction is defined by the above scaling in the limit of $\epsilon \rightarrow 0$. For $n=0$ this has been studied in [33] where it was shown that the resultant contracted algebra admits an infinite dimensional extension containing one copy of Virasoro algebra. In what follows we would like to extend this consideration for general $n$.

\section{$2.1 \quad$ Field theory description}

We start from a CFT in $d+1$ dimensions. The theory is invariant under the action of generators of conformal algebra given by rotations $J_{\mu \nu}$, translations $P_{\mu}$, dilation $D$ and

\footnotetext{
${ }^{1}$ We note, however, that calling this theory a non-relativistic CFT is somehow misleading as it has relativistic properties in those directions which remained unchanged
} 
special conformal transformations $K_{\mu}$ whose representations as a vector field acting on the $d+1$ dimensional Minkowski space are given by

$$
J_{\mu \nu}=-\left(x_{\mu} \partial_{\nu}-x_{\nu} \partial_{\mu}\right), \quad P_{\mu}=\partial_{\mu}, \quad D=-(x \cdot \partial), \quad K_{\mu}=-\left(2 x_{\mu}(x \cdot \partial)-(x \cdot x) \partial_{\mu}\right)
$$

with $\mu=0, \cdots, d$. The aim is to contract the conformal algebra generated by the above generators. To do this we will consider the general scaling (2.1) in the limit of $\epsilon \rightarrow 0$. To be specific we will consider the case of $n=1$. It is, of course, straightforward to generalize it for arbitrary $n$.

For $n=0$ under the above rescaling and in the of $\epsilon \rightarrow 0$ the $d+1$ dimensional conformal algebra reduces to Galilean conformal algebra studied in [33]. For $n=1$ the generators of the corresponding algebra as a vector field acting on a $d+1$ dimensional Minkowski space ( for $d \geq 2$ ) are given by

$$
\begin{aligned}
& J_{i j}=-\left(x_{i} \partial_{j}-x_{j} \partial_{i}\right), \quad J_{i}=t \partial_{i}, \quad \tilde{J}_{i}=-y \partial_{i}, \quad P_{i}=\partial_{i}, \quad K_{i}=\left(y^{2}-t^{2}\right) \partial_{i}, \\
& D=-\left(x_{i} \partial_{i}+y \partial_{y}+t \partial_{t}\right), \quad \tilde{D}=t \partial_{y}+y \partial_{t}, \quad P=\partial_{t}, \quad \tilde{P}=\partial_{y}, \\
& K=\left(t^{2}+y^{2}\right) \partial_{t}+2 t y \partial_{y}+2 t x_{i} \partial_{i}, \quad \tilde{K}=-\left(t^{2}+y^{2}\right) \partial_{y}-2 t y \partial_{t}-2 y x_{i} \partial_{i} .
\end{aligned}
$$

To have an insight how the semi-Galilean conformal algebra for $n=1$ could be, it is useful to define new coordinates $u=t+y, v=t-y$ by which the above generators may be recast to the following form 2

$$
\begin{aligned}
& H=2 \partial_{u}, \quad E=2\left(u \partial_{u}+\frac{1}{2} x_{i} \partial_{i}\right), \quad C=2\left(u^{2} \partial_{u}+u x_{i} \partial_{i}\right), \\
& \bar{H}=2 \partial_{v}, \quad \bar{E}=-2\left(v \partial_{v}+\frac{1}{2} x_{i} \partial_{i}\right), \quad \bar{C}=2\left(v^{2} \partial_{v}+v x_{i} \partial_{i}\right), \\
& J_{i j}=-\left(x_{i} \partial_{j}-x_{j} \partial_{i}\right), \quad P_{i}=\partial_{i}, \quad B_{i}=-u \partial_{i}, \quad \bar{B}_{i}=v \partial_{i}, \quad K_{i}=-u v \partial_{i} .
\end{aligned}
$$

It is easy to see that $(H, E, C)$ and $(\bar{H}, \bar{E}, \bar{C})$ generate two copies of $S L(2, R)$ algebra. In fact to write the explicit form of the commutation relations of the algebra it is useful to define $L_{ \pm 1,0}, \bar{L}_{ \pm 1,0}$ and $M_{i r s} r, s=0,1$ as follows

$$
\begin{aligned}
& \left\{L_{-1}=\frac{1}{2} H, \quad L_{0}=\frac{1}{2} E, \quad L_{1}=\frac{1}{2} C\right\}, \quad\left\{\bar{L}_{-1}=\frac{1}{2} \bar{H}, \quad \bar{L}_{0}=-\frac{1}{2} \bar{E}, \bar{L}_{1}=\frac{1}{2} \bar{C}\right\}, \\
& \left\{M_{i 00}=-P_{i}, \quad M_{i 01}=B_{i}, \quad M_{i 10}=-\bar{B}_{i}, \quad M_{i 11}=K_{i}\right\} .
\end{aligned}
$$

Using this notation the non-zero commutation relations of the algebra are

$$
\begin{array}{ll}
{\left[L_{n}, L_{m}\right]=(n-m) L_{n+m},} & {\left[\bar{L}_{n}, \bar{L}_{m}\right]=(n-m) \bar{L}_{n+m},} \\
{\left[L_{n}, M_{i r s}\right]=\left(\frac{n+1}{2}-r\right) M_{i(n+r) s},} & {\left[\bar{L}_{n}, M_{i r s}\right]=\left(\frac{n+1}{2}-s\right) M_{i s(n+s)},}
\end{array}
$$

\footnotetext{
${ }^{2}$ For example $H=\tilde{P}+P, C=K-\tilde{K}$ and $E=\tilde{D}-D$.
} 


$$
\left[M_{l r s}, J_{i j}\right]=\left(\delta_{j l} M_{i r s}-\delta_{i l} M_{j r s}\right), \quad\left[J_{i j}, J_{i^{\prime} j^{\prime}}\right]=s o(d-1),
$$

which make the two $S L(2, R)$ subalgebras manifest. Here $n, m= \pm 1,0$ and $r, s=0,1$. Actually it is useful to re-express the generators of $(2.5)$ in the following instructive closed form

$$
L_{n}=u^{n+1} \partial_{u}+\frac{n+1}{2} u^{n} x_{i} \partial_{i}, \quad \bar{L}_{n}=v^{n+1} \partial_{v}+\frac{n+1}{2} v^{n} x_{i} \partial_{i}, \quad M_{i r s}=-u^{r} v^{s} \partial_{i} .
$$

From these expressions it is natural to define the above vector fields for arbitrary integers $n$ and $r$. Indeed defining

$$
J_{i j n m}=-u^{n} v^{m}\left(x_{i} \partial_{j}-x_{j} \partial_{i}\right),
$$

one finds an infinite dimensional algebra as follows

$$
\begin{array}{ll}
{\left[L_{n}, L_{m}\right]=(n-m) L_{n+m},} & {\left[\bar{L}_{n}, \bar{L}_{m}\right]=(n-m) \bar{L}_{n+m},} \\
{\left[M_{i n m}, M_{j n^{\prime} m^{\prime}}\right]=0,} & {\left[L_{n}, \bar{L}_{m}\right]=0,} \\
{\left[L_{n}, M_{i m l}\right]=\left(\frac{n+1}{2}-m\right) M_{i(n+m) l},} & {\left[\bar{L}_{n}, M_{i m l}\right]=\left(\frac{n+1}{2}-l\right) M_{i m(n+l)}} \\
{\left[L_{n}, J_{i j m l}\right]=-m J_{i j(n+m) l},} & {\left[\bar{L}_{n}, J_{i j m l}\right]=-l J_{i j m(n+l)}} \\
{\left[M_{l n m}, J_{i j n^{\prime} m^{\prime}}\right]=\left(\delta_{j l} M_{i\left(n+n^{\prime}\right)\left(m+m^{\prime}\right)}-\delta_{i l} M_{j\left(n+n^{\prime}\right)\left(m+m^{\prime}\right)}\right) .}
\end{array}
$$

Moreover the $J_{i j n m}$ 's generate an $s o(d-1)$ affine algebra.

As an conclusion we observed that the (semi) Galilean conformal algebra obtained from the relativistic conformal algebra using the contraction (2.1) admits an infinite dimensional extension for $n=0,1$. This may be understood from the fact that in these cases there is at least an $S L(2, R)$ subalgebra which may be extended to a Virasoro algebra. As we will see from gravity point of view this corresponds to the fact that in these cases the gravity dual develops $A d S_{2}$ or $A d S_{3}$ geometries for $n=0$ and $n=1$, respectively.

Actually the above procedure may be generalized for $n \geq 2$ where we will get an algebra containing an $s o(2, n+1) \times s o(d-n-1)$ subalgebra. We note, however, that in this case the resultant semi-Galilean algebra does not admit an infinite dimensional extension. As we will see in the next section the reason may be understood from the fact that in this case the gravity background develops an $A d S_{n+2}$ geometry which has finite dimensional asymptotic symmetry algebra.

\subsection{Gravity description}

Following AdS/CFT correspondence we would expect that a $d+1$ dimensional CFT may have a gravity dual defined on a background containing an $A d S_{d+2}$ factor where the CFT lives on the boundary of the $A d S$ space. Therefore one should be able to take the nonrelativistic limit from both side of the duality. In particular we would like to carry out the contraction of the previous section on the AdS part of the bulk geometry. 
To proceed consider the metric of an $A d S_{d+2}$ space in the Poincaré coordinates

$$
d s^{2}=\frac{-d t^{2}+d y_{\alpha}^{2}+d x_{i}^{2}+d z^{2}}{z^{2}} .
$$

The non-relativistic limit of the previous section can be generalized to the bulk geometry as follows

$$
t \rightarrow t, \quad z \rightarrow z, \quad y_{\alpha} \rightarrow y_{\alpha}, \quad x_{i} \rightarrow \epsilon x_{i} .
$$

In the limit of $\epsilon \rightarrow 0$ where only $t, z$ and $y_{\alpha}$ survive the contraction the resultant geometry develops an $A d S_{n+2}$ space. The rest $d-n$ dimensional space parametrized by $x_{i}$ are fibered over the $A d S_{n+2}$ base spacetime. Indeed as it was argued in [33] the corresponding gravity dual should be given in terms of the Newton-Cartan like description where $A d S_{n+2}$ plays the special role of the time. In this formalism the metric is non-dynamical and the dynamics are given by torsion free affine connections. More precisely following [33] one may define a contravariant tensor $\gamma=\gamma^{M N} \partial_{M} \otimes \partial_{N}$ with $M, N=\{t, z, \alpha, i\}$. It has $n+2$ zero eigenvalues corresponding to $\left\{t, z, y_{\alpha}\right\}$ which parametrize the base $A d S_{n+2}$ space with the metric

$$
d s^{2}=g_{a b} d x^{a} d x^{b}=\frac{-d t^{2}+d y_{\alpha}^{2}+d z^{2}}{z^{2}} .
$$

The affine connections $\Gamma_{N L}^{M}$ are compatible with both base AdS space as well as the $d-n$ dimensional spatial fiber

$$
\nabla_{M} \gamma^{M L}=0, \quad \nabla_{M} g_{a b}=0 .
$$

In our case the dynamical connection may be given by $\Gamma_{a b}^{i}=\partial_{i} \Phi_{a b}[33]$.

Following the general lore of the AdS/CFT correspondence [49] we would expect that if the above Newtonian gravity provides a gravity dual of the semi-Galilean conformal filed theory one should be able to see the semi-Galilean symmetry algebra as the asymptotic symmetry algebra of the above geometry in the sense of Brown and Henneaux construction [50]. In what follows we will show that this is, indeed, the case. To be specific we will consider the case of $n=1$ which turns out to be more interesting case. Generalization to other cases is straightforward.

In the Poincaré coordinates the Killing vectors of $A d S_{d+2}$ are given by

$$
\begin{array}{rr}
J_{\mu \nu}=-\left(x_{\mu} \partial_{\nu}-x_{\nu} \partial_{\mu}\right), & D=-\left(x^{\mu} \partial_{\mu}+z \partial_{z}\right), \\
K_{\mu}=-\left(2 x_{\mu}\left(x^{\nu} \partial_{\nu}+z \partial_{z}\right)-\left(x^{\nu} x_{\nu}+z^{2}\right) \partial_{\mu}\right), & P_{\mu}=\partial_{\mu} .
\end{array}
$$

Using the scaling (2.11) the resultant contracted Killing vectors read

$$
\begin{aligned}
& P_{i}=\partial_{i}, \quad B_{i}=t \partial_{i}, \quad \tilde{B}_{i}=-y \partial_{i}, \quad K_{i}=\left(t^{2}-y^{2}-z^{2}\right) \partial_{i}, \\
& D=-\left(t \partial_{t}+y \partial_{y}+x_{i} \partial_{i}+z \partial_{z}\right), \quad \tilde{D}=t \partial_{y}+y \partial_{t}, \quad J_{i j}=-\left(x_{i} \partial_{j}-x_{j} \partial_{i}\right), \\
& K=-\left(t^{2}+y^{2}+z^{2}\right) \partial_{t}-2 z t \partial_{z}-2 t y \partial_{y}-2 t x_{i} \partial_{i}, \quad P=\partial_{t}, \\
& \tilde{K}=\left(t^{2}+y^{2}-z^{2}\right) \partial_{y}+2 t y \partial_{t}+2 z y \partial_{z}+2 y x_{i} \partial_{i}, \quad \tilde{P}=\partial_{y} .
\end{aligned}
$$


Note that to make the comparison more transparent we have used the same labeling for bulk and boundary generators. Following our previous discussions setting $u=t+y, v=t-y$ the above Killing vectors may be recast to the following form

$$
\begin{aligned}
& H=2 \partial_{u}, \quad E=2\left(u \partial_{u}+\frac{1}{2} x_{i} \partial_{i}+\frac{1}{2} z \partial_{z}\right), \quad C=2\left(u^{2} \partial_{u}+u\left(x_{i} \partial_{i}+z \partial_{z}\right)+z^{2} \partial_{v}\right), \\
& \bar{H}=2 \partial_{v}, \quad \bar{E}=-2\left(v \partial_{v}+\frac{1}{2} x_{i} \partial_{i}+\frac{1}{2} z \partial_{z}\right), \quad \bar{C}=-2\left(v^{2} \partial_{v}+v\left(x_{i} \partial_{i}+z \partial_{z}\right)+z^{2} \partial_{u}\right), \\
& J_{i j}=-\left(x_{i} \partial_{j}-x_{j} \partial_{i}\right), \quad P_{i}=\partial_{i}, \quad B_{i}=u \partial_{i}, \quad \bar{B}_{i}=v \partial_{i}, \quad K_{i}=\left(u v-z^{2}\right) \partial_{i} .
\end{aligned}
$$

which reduce to those in the previous section in the limit of $z \rightarrow 0$ where we approach the boundary of the $A d S_{3}$.

Let us define infinite dimensional vector fields in the bulk as follows

$$
\begin{aligned}
& L_{n}=u^{n+1} \partial_{u}+\frac{n+1}{2} u^{n}\left(x_{i} \partial_{i}+z \partial_{z}\right)+\frac{n(n+1)}{2} u^{n-1} z^{2} \partial_{v}, \\
& \bar{L}_{n}=v^{n+1} \partial_{v}+\frac{n+1}{2} v^{n}\left(x_{i} \partial_{i}+z \partial_{z}\right)+\frac{n(n+1)}{2} v^{n-1} z^{2} \partial_{u}
\end{aligned}
$$

which can be properly identified with $H, E, C$ and $\bar{H}, \bar{E}, \bar{C}$ for $n= \pm 1,0$ which at the boundary where $z \rightarrow 0$ reduce to those in (2.7). It is interesting to note that these vector fields asymptotically obey two copies of Virasoro algebra, i.e.

$$
\left[L_{n}, L_{m}\right]=(n-m) L_{n+m}, \quad\left[\bar{L}_{n}, \bar{L}_{m}\right]=(n-m) \bar{L}_{n+m}, \quad\left[\bar{L}_{n}, L_{m}\right]=\mathcal{O}\left(z^{4}\right) .
$$

The action of the Virasoro generators on the metric of the base manifold, $A d S_{3}$, is given

$$
L_{n}: d s^{2} \rightarrow d s^{2}+\frac{n\left(n^{2}-1\right)}{2} u^{n-2} d u^{2}, \quad \bar{L}_{n}: d s^{2} \rightarrow d s^{2}+\frac{n\left(n^{2}-1\right)}{2} v^{n-2} d v^{2} .
$$

Therefore the generators of two $S L(2, R)$ 's given by $L_{ \pm, 0}$ and $\bar{L}_{ \pm, 0}$ are the exact isometries of the base metric, as expected, while for other $n$ 's they generate the asymptotic symmetry which preserve the following boundary conditions

$$
\left(\begin{array}{lll}
h_{u u}=\mathcal{O}(1) & h_{u v}=\mathcal{O}(1) & h_{u z}=\mathcal{O}(z) \\
h_{v u}=h_{u v} & h_{v v}=\mathcal{O}(1) & h_{v z}=\mathcal{O}(z) \\
h_{z u}=h_{u z} & h_{z v}=h_{v z} & h_{z z}=\mathcal{O}(1)
\end{array}\right)
$$

On the other hand requiring to have asymptotically a closed algebra we will have to extend the other generators as follows

$$
M_{i n m}=-\left(u^{n} v^{m}-n m u^{n-1} v^{m-1} z^{2}\right) \partial_{i}, \quad J_{i j n m}=-u^{n} v^{m}\left(x_{i} \partial_{j}-x_{j} \partial_{i}\right),
$$

which for $m, n=0,1$ can be identified with $P_{i}, B_{i}, \bar{B}_{i}, K_{i}$ and at the boundary where $z \rightarrow 0$ reduce to that in (2.7). It is easy to see that

$$
\left[L_{n}, M_{i l m}\right]=\left(\frac{n+1}{2}-l\right) M_{i(n+l) m}+\mathcal{O}\left(z^{4}\right), \quad\left[L_{n}, J_{i j l m}\right]=-l J_{i j(n+l) m}+\mathcal{O}\left(z^{2}\right),
$$




$$
\begin{aligned}
& {\left[\bar{L}_{n}, M_{i l m}\right]=\left(\frac{n+1}{2}-m\right) M_{i l(n+m)}+\mathcal{O}\left(z^{4}\right), \quad\left[\bar{L}_{n}, J_{i j l m}\right]=-m J_{i j l(n+m)}+\mathcal{O}\left(z^{2}\right),} \\
& {\left[M_{l n m}, J_{i j n^{\prime} m^{\prime}}\right]=\left(\delta_{j l} M_{i\left(n+n^{\prime}\right)\left(m+m^{\prime}\right)}-\delta_{i l} M_{j\left(n+n^{\prime}\right)\left(m+m^{\prime}\right)}\right)+\mathcal{O}\left(z^{2}\right)} \\
& {\left[M_{i n m}, M_{j n^{\prime} m^{\prime}}\right]=0 .}
\end{aligned}
$$

As a conclusion we have demonstrated that the asymptotic symmetry algebra of our bulk geometry is the semi-Galilean conformal algebra studied in the previous section.

It is straightforward to generalize the above considerations for $n \geq 2$ where the base space will be $A d S_{n+2}$. In this case from the base space we find an $s o(2, n+1)$ factor while from the fiber one gets an $s o(d-n-1)$ subalgebra which is compatible with those studies in the previous section. We note that for the case of $n \geq 2$ the semi-Galilean conformal algebra is finite dimensional due to the fact the the asymptotic symmetry of $A d S_{n+2}$ for $n \geq 2$ is finite dimensional.

\section{AdS/CFT description of theory with Galilean con- formal symmetry}

In this section we would like to study the AdS/CFT correspondence for the Galilean conformal field theory. Following the relativistic CFT one would expect that in the Galilean CFT the asymptotic states cannot be defined and the physical observables would be correlation functions. Therefore the task is to compute $N$-point functions of operators in the Galilean CFT which is the aim of this section.

In what follows we will mainly consider the case of $n=0$, though the procedure may be generalized for other $n$ 's.

\subsection{Field theory description}

Consider a Galilean CFT in $d+1$ dimensions. As we have seen the corresponding algebra can be obtained from the relativistic CFT by a contraction. Therefore one may naively expect that the $N$-point functions of Galilean CFT can also be obtained from those in the relativistic CFT by making use of the same contraction. For example consider two point function of an operator $\phi$ with scaling dimension $\Delta$ in a relativistic CFT in $d+1$ dimensions parametrized by $t$ and $x_{i}$

$$
\left\langle\phi\left(t_{1}, x_{i}\right) \phi\left(t_{2}, y_{i}\right)\right\rangle \sim \frac{1}{\left(-\left(t_{1}-t_{2}\right)^{2}+\left(x_{i}-y_{i}\right)^{2}\right)^{\Delta}} .
$$

Using the scaling limit (2.1) and in the limit of $\epsilon \rightarrow 0$ the two point function of the Galilean CFT reads

$$
\left\langle\phi\left(t_{1}, x_{i}\right) \phi\left(t_{2}, y_{i}\right)\right\rangle \sim \frac{1}{\left(t_{1}-t_{2}\right)^{2 \Delta}} .
$$

Similarly we can extend the above procedure to $N$-point function to conclude that in general the $N$-point function of Galilean CFT depends only on time. 
We, note, however that although the above results seem reasonable, the way we reach the conclusion may not be correct in general. The reason is due to the fact that the representations of an algebra under a contraction do not necessarily lead to faithful (bijective) representations [51]. In other words although the $N$-point functions we obtain by this method satisfy the Ward identity of the Galilean CFT, it is not clear that the general form of the $N$-point functions can be obtained from this method. Therefore it would be interesting to evaluate the $N$-point function of Galilean CFT directly. To do this we utilize the Ward identity of the Galilean CFT.

The representation of the generators of the Galilean conformal algebra acting on an operator with dimension $\Delta$ is given

$$
\begin{aligned}
& J_{i j}=-\left(x_{i} p_{j}-x_{j} p_{i}\right), \quad P_{0}=H=-\partial_{t}, \quad P_{i}=\partial_{i} \quad B_{i}=t \partial_{i}, \\
& D=-\left(x_{i} \partial_{i}+t \partial_{t}\right)-\Delta, \quad K=-\left(2 t x_{i} \partial_{i}+t^{2} \partial_{t}\right)-2 \Delta t, \quad K_{i}=t^{2} \partial_{i}
\end{aligned}
$$

Note that although the Galilean conformal algebra admits an infinite dimensional extension, we would expect that the vacuum is only invariant under the global part of the algebra given by the above generators. Therefore the Wrad identity of the Galilean CFT may be written as follows

$$
\sum_{i}\left\langle 0\left|\phi\left(x_{1}\right) \ldots Q \phi\left(x_{i}\right) \ldots \phi\left(x_{N}\right)\right| 0\right\rangle=0
$$

where $|0\rangle$ is a vacuum which is invariant under the global part of the algebra. $Q \phi\left(x_{i}\right)$ is the representation of an operator $Q$ on the field $\phi\left(x_{i}\right)$ with $Q$ stands for one of the generators in (3.3). By making use of the equation (3.4) one can write the Ward identities for $N$ point function $G_{N}\left(x_{1}, t_{1}, \ldots, x_{N}, t_{N}\right)$. To write the explicit form of the Wrad identities for $N$-point functions it is useful to define new variables $t_{i 2}=t_{i}-t_{2}, x_{i 2}=x_{i}-x_{2}$ and $\tilde{t}_{12}=t_{1}+t_{2}, \tilde{x}_{12}=x_{1}+x_{2}$ for $i=1,3,4 \cdots, N$.

From the Ward identities for space and time translations one finds that $G_{N}$ depends only on $t_{i 2}$ and $x_{i 2}$, i.e. $G_{N}\left(t_{12}, t_{32}, \cdots ; x_{12}, x_{32}, \cdots\right)$. On the other hand from $K_{i}$ one finds

$$
\begin{aligned}
& {\left[t_{12} D_{12}+t_{32} D_{32}+\ldots+t_{N 2} D_{N 2}\right] G_{N}=0} \\
& {\left[t_{32}\left(t_{32}-t_{12}\right) D_{32}+\ldots+t_{N 2}\left(t_{N 2}-t_{12}\right) D_{N 2}\right] G_{N}=0}
\end{aligned}
$$

where $D_{i 2}=\frac{\partial}{\partial x_{i 2}}$. From the dilatation we get

$$
\left[x_{12} D_{12}+\ldots+x_{N 2} D_{N 2}+t_{12} \partial_{12}+\ldots+t_{N 2} \partial_{N 2}+\lambda_{1}+\ldots+\lambda_{N}\right] G_{N}=0,
$$

and $K$ leads to the following differential equation

$$
\begin{aligned}
& {\left[\left(2 t_{32} x_{32}-t_{32} x_{12}-t_{12} x_{32}\right) D_{32}+\ldots+\left(2 t_{N 2} x_{N 2}-t_{N 2} x_{12}-t_{12} x_{N 2}\right) D_{N 2}\right.} \\
& +t_{32}\left(t_{32}-t_{12}\right) \partial_{32}+\ldots+t_{N 2}\left(t_{N 2}-t_{12}\right) \partial_{N 2}+t_{12}\left(\lambda_{1}-\lambda_{2}-\ldots-\lambda_{N}\right) \\
& \left.+2 \lambda_{3} t_{32}+\ldots+2 \lambda_{N} t_{N}\right] G_{N}=0 .
\end{aligned}
$$

Now the task is to solve these equations to find $N$-point functions. We note, however, that these equations cannot fix the $N$-point functions completely for arbitrary $N$. This is of course the case even for relativistic one where the $N$-point functions can be found up to unknown functions. Let us give the explicit form of two and there point functions. 


\section{Two-point function}

From (3.5) it is clear that the two-point function does not depend on $x_{12}$ and from (3.6) we get

$$
G_{2}:=\left\langle\phi_{1}\left(t_{1}, x_{i 1}\right) \phi_{2}\left(t_{2}, x_{i 2}\right)\right\rangle=C t_{12}^{-\Delta}, \quad \Delta=\Delta_{1}+\Delta_{2}
$$

where $C$ is a constant.

\section{Three-point function}

From (3.5) it is clear that the three-point function does not depend on $x_{12}$ and $x_{32}$ and from (3.6) and (3.7) one finds

$$
G_{3}:=\left\langle\phi_{1}\left(t_{1}, x_{i 1}\right) \phi_{2}\left(t_{2}, x_{i 2}\right) \phi_{3}\left(t_{3}, x_{i 3}\right)\right\rangle=C\left(\frac{1}{t_{12}}\right)^{\Delta_{1}+\Delta_{2}-\Delta_{3}}\left(\frac{1}{t_{32}}\right)^{-\Delta_{1}+\Delta_{2}+\Delta_{3}}\left(\frac{1}{t_{13}}\right)^{\Delta_{1}+\Delta_{3}-\Delta_{2}}
$$

\section{$N$-point function}

In principle one could proceed to compute $N$-point function for arbitrary $N$, though here we will not do that. The only comment we would like to make is that utilizing the Ward identities one can show that the $N$-point function depends only on $t_{i 2}$ 's.

\subsection{Gravity description}

In this subsection we would like to see how the $N$-point functions we have considered in the previous section can be obtained from gravity description. The procedure in the relativistic AdS/CFT correspondence is to evaluate the bulk action on a classical solution with a given boundary condition. Since for Galilean CFT the gravity description is given in terms of the Newtonian gravity the above description may not be directly applied in this case. To explore the procedure we start from a propagating field on an AdS geometry and impose the contraction we have introduced in the previous section.

To proceed, for simplicity, we consider a massive scalar field on the $A d S_{d+2}$ background whose equation of motion is given by

$$
\frac{1}{\sqrt{G}} \partial_{M}\left(\sqrt{G} G^{M N} \partial_{N} \phi\left(t, z, x_{i}\right)\right)-m^{2} \phi\left(t, z, x_{i}\right)=0
$$

where $G_{M N}$ is the metric of the AdS geometry. To be specific we consider the AdS geometry in the Poincaré coordinates parametrized by $t, z, x_{i}$. Under the scaling (2.11) one has

$$
G_{M M} \rightarrow G_{M N}, \quad \partial_{t} \rightarrow \partial_{t}, \quad \partial_{z} \rightarrow \partial_{z}, \quad \partial_{i} \rightarrow \epsilon^{-1} \partial_{i} .
$$

so that

$$
\left[\frac{1}{\sqrt{G}} \partial_{a}\left(\sqrt{G} g^{a b} \partial_{b} \phi\left(t, z, x_{i}\right)\right)-m^{2} \phi\left(t, z, x_{i}\right)\right]+\frac{z^{2}}{\epsilon^{2}} \partial_{i}^{2} \phi\left(t, z, x_{i}\right)=0
$$


Here $g_{a b}$ is the metric of $A d S_{2}$ base geometry. In order to have a well behaved equation in the limit of $\epsilon \rightarrow 0$ one should impose

$$
\frac{1}{\sqrt{G}} \partial_{a}\left(\sqrt{G} g^{a b} \partial_{b} \phi\left(t, z, x_{i}\right)\right)-m^{2} \phi\left(t, z, x_{i}\right)=0, \quad \partial_{i}^{2} \phi\left(t, z, x_{i}\right)=0 .
$$

The first equation may be obtained from a two dimensional action given by

$$
I=\int d t d z \sqrt{G} \frac{1}{2}\left(g^{a b} \partial_{a} \phi \partial_{b} \phi+m^{2} \phi^{2}\right)
$$

while the second equation may be treated as a constraint. Therefore the most general solution of the equation of motion of the above action is

$$
\phi(t, z)=z^{\frac{d+1}{2}} e^{-i \omega t}\left(A I_{\alpha}(\omega z)+B K_{\alpha}(\omega z)\right)
$$

where $\alpha=\sqrt{\frac{(d+1)^{2}}{4}+m^{2}}$. Since in the present case the constraint decouples from the equation of motion, it leads to an overall factor which could depends on $x \sqrt[3]{3}$. It is then straightforward to follow the general role of the AdS/CFT correspondence to find the bulk solution by given a boundary value as follows

$$
\phi(t, z)=c \delta^{\Delta-d-1} \int d t^{\prime} \phi_{\delta}\left(t^{\prime}\right)\left(\frac{z}{z^{2}+\left|t-t^{\prime}\right|^{2}}\right)^{\Delta}
$$

where $\Delta=\frac{d}{2}+\alpha$ and $\phi_{\delta}$ denotes the Dirichlet boundary value at $z=\delta$. This can be used to read the two point function as follows

$$
\left\langle\mathcal{O}\left(t_{1}\right) \mathcal{O}\left(t_{2}\right)\right\rangle \sim \frac{1}{\left(t_{1}-t_{2}\right)^{2 \Delta}}
$$

in agreement with (3.8) for $\Delta_{1}=\Delta_{2}=\Delta$.

To find $N$-point function we should add an interaction term $\lambda_{N} \phi^{N}$ to the action. Then following the standard AdS/CFT procedure one arrives at (see for example [52])

$$
I_{N}\left(t_{1}, \ldots, t_{N}\right) \sim \int d t d z \frac{z^{-(d+2)+N \Delta}}{\left[\left(z^{2}+\left(t-t_{1}\right)^{2}\right) \ldots\left(z^{2}+\left(t-t_{N}\right)^{2}\right)\right]^{\Delta}} .
$$

In particular for $N=3$ we get

$$
\left\langle\mathcal{O}\left(t_{1}\right) \mathcal{O}\left(t_{2}\right) \mathcal{O}\left(t_{3}\right)\right\rangle \sim-\frac{\lambda_{3} \Gamma\left(\frac{1}{2} \Delta+\alpha\right)}{2 \pi^{d+1}}\left[\frac{\Gamma\left(\frac{1}{2} \Delta\right)}{\Gamma(\alpha)}\right]^{3} \frac{1}{\left(t_{12} t_{31} t_{23}\right)^{\Delta}}
$$

in agreement with (3.9) for $\Delta_{1}=\Delta_{2}=\Delta_{3}=\Delta$.

\footnotetext{
${ }^{3}$ We note, however, that the over all factor could parametrically be divergent due to the integration over boundary term. This might be observed by redefinition of the boundary operators by making use of a regularization. The similar behavior happens in the non-relativistic CFT studied in $[2,32]$.
} 
As a conclusion we have demonstrated how $N$-point function can be obtained from gravity description of Galilean CFT where we have seen that the main role plays by the base $A d S_{2}$ geometry.

Actually the procedure may be summarized as follow. In order to obtain the physical correlation functions one needs to use the standard AdS/CFT correspondence for $A d S_{2}$ part, though the action of the corresponding propagating fields in the $A d S_{2}$ geometry gets a contribution from the fiber via the measure of the integral. Otherwise the procedure follows the same as that in the standard AdS/CFT correspondence applied for $A d S_{2}$.

The above procedure may be generalized to arbitrary $n$. The only difference is that the $A d S_{2}$ has to be replaced by $A d S_{n+2}$. In other words in this case the action is given by

$$
I=\int d t d z d^{n} y_{\alpha} z^{d-n-1} \sqrt{g} \frac{1}{2}\left(g^{a b} \partial_{a} \phi \partial_{b} \phi+m^{2} \phi^{2}\right)
$$

where $g_{a b}$ is the metric of $A d S_{n+2}$ given by (2.12). Note that the propagating fields are subject to the constraint $\partial_{i}^{2} \phi=0$ for $i=1, \cdots, d-n-1$.

It is important to note that the decoupling of the fiber is due to the particular form of the constraint. If we change the constraint (for example by breaking the conformal symmetry via heating up the theory) the situation may be changed.

\section{Discussions}

In this paper we have considered different contractions of a $d+1$ dimensional relativistic conformal algebra. The contraction is defined by the scaling (2.1) in the limit of $\epsilon \rightarrow 0$. In other words if we define the velocity of $l$ th direction as

$$
v_{\alpha}=\frac{y_{\alpha}}{t}, \quad v_{i}=\frac{x_{i}}{t}, \quad \alpha=1, \cdots, n, \quad i=n+1, \cdots, d,
$$

in this limit one has $v_{i} \rightarrow 0$. Therefore the contraction may be thought of as taking nonrelativistic limit of the relativistic conformal algebra. In particular when $n=0$ the resultant algebra is the Galilean conformal algebra [33]. For $n \geq 1$ we are taking non-relativistic limit in some directions while the others remain unchanged. So, it may be treated as a semi-Galilean conformal algebra.

In general by a contraction the conformal algebra in $d+1$ dimension, $s o(2, d+1)$, reduces to an algebra which contains an $s o(2, n+1) \times s o(d-n-1)$ subalgebra. For $n=0$ and $n=1$ the obtained algebras have $S L(2, R)$ and $S L(2, R) \times S L(2, R)$ subalgebra, respectively. Due to this property the corresponding algebras have infinite dimensional extension where the $S L(2, R)$ 's extend to the Virasoro algebra. Having had the Virasoro algebra in the cases of $n=0,1$, it would be interesting to see if the (semi) Galilean conformal algebra allows a central extension to its Virasoro subalgebra.

Following the AdS/CFT correspondence one may suspect that the (semi) Galilean CFTs may have dual gravity descriptions. If so, the corresponding gravity dual should contain a factor of $A d S_{n+2}$ to support the symmetry group $S O(2, n+1)$. Moreover to have the symmetry group $S O(d-n-1)$ the gravity dual should also have a factor of $d-n-$

1 dimensional flat space, $\mathcal{M}_{d-n-1}$. On the other hand since the semi-Galilean conformal 
algebra cannot be factorized as $s o(2, n+1) \times s o(d-n-1)$ the bulk geometry is not a direct product of these two spaces, though locally it may be thought of as $A d S_{n+2} \times \mathcal{M}_{d-n-1}$. In fact it was argued in [33] that at least for $n=0$ the geometry is a $d-1$ dimensional spatial space fibered over an $A d S_{2}$. From our consideration we expect that in general the bulk geometry is a $d-n-1$ dimensional spatial space fibered over an $A d S_{n+2}$. Note, that, the corresponding gravity is given in terms of Newton-Cartan like description when the role of time is replaced by an $A d S_{n+2}$.

Using this picture it is easy to understand why the cases of $n=0$ and 1 have infinite dimensional extension while the other cases are finite dimensional. In fact the reason is due to the asymptotic symmetry of AdS space; while for $A d S_{2}$ and $A d S_{3}$ it is infinite dimensional, for the others it is finite dimensional 4 .

We have also explored the AdS/CFT correspondence for (semi) Galilean CFTs where we have seen that the essential role is played by the base $A d S_{n+2}$ geometry. In fact the correlation functions of the (semi) Galilean CFTs can be evaluated by making use of propagating fields on $A d S_{n+2}$ with proper boundary conditions and a modified measure due to the contribution of the fiber.

An interesting application of this contraction would be to apply the procedure to $\mathcal{N}=4$ four dimensional SYM theory whose gravity dual is given by type IIB string theory on $A d S_{5} \times S^{5}$. Taking the limit from both sides of the duality one may single out a subset of $\mathcal{N}=4$ four dimensional SYM theory which has (semi) Galilean conformal symmetry. This might give a new insight about the AdS/CFT correspondence following [53].

In the context of AdS/CFT duality it is known that heating up the dual field theory generically corresponds to adding a black hole in the bulk gravity. Therefore we would expect that applying the above limit one may find a gravity dual to the (semi) Galilean CFT at finite temperature. In this case the bulk gravity background may be interpreted as a $d-n-1$ dimensional spatial space fibered over a base which is given by a black hole in $A d S_{n+2}$ space. It is worth noting that in this case the contraction may by supplemented by a shift in $\partial_{t}$. In this case the constraint does not decouple from the equation of motion of propagating modes in the base $A d S_{n+2}$ space. As a result the correlation function will depend on the fiber coordinates too [54].

\section{Acknowledgments}

We would also like to thank Hamid Afshar, Amin Akhavan, Davod Allahbakhsi, Reza Fareghbal, Amir E. Mosaffa and Shahin Rouhani for discussions on the different aspects of nonrelativistic AdS/CFT correspondence. This work is supported in part by Iranian TWAS chapter at ISMO.

\footnotetext{
${ }^{4}$ It should be compared with Schrödinger algebra which has infinite dimensional extension in any dimension.
} 


\section{References}

[1] D. T. Son, "Toward an AdS/cold atoms correspondence: a geometric realization of the Schroedinger symmetry," Phys. Rev. D 78, 046003 (2008) arXiv:0804.3972 [hep-th]].

[2] K. Balasubramanian and J. McGreevy, "Gravity duals for non-relativistic CFTs," Phys. Rev. Lett. 101, 061601 (2008) [arXiv:0804.4053 [hep-th]].

[3] W. D. Goldberger, "AdS/CFT duality for non-relativistic field theory," arXiv:0806.2867 [hep-th].

[4] J. L. B. Barbon and C. A. Fuertes, "On the spectrum of nonrelativistic AdS/CFT," JHEP 0809, 030 (2008) arXiv:0806.3244 [hep-th]].

[5] C. P. Herzog, M. Rangamani and S. F. Ross, "Heating up Galilean holography," arXiv:0807.1099 [hep-th].

[6] J. Maldacena, D. Martelli and Y. Tachikawa, "Comments on string theory backgrounds with non-relativistic conformal symmetry," JHEP 0810, 072 (2008) arXiv:0807.1100 [hep-th]].

[7] A. Adams, K. Balasubramanian and J. McGreevy, "Hot Spacetimes for Cold Atoms," arXiv:0807.1111 [hep-th].

[8] C. Leiva and M. S. Plyushchay, "Conformal symmetry of relativistic and nonrelativistic systems and AdS/CFT correspondence," Annals Phys. 307, 372 (2003) arXiv:hep-th/0301244.

[9] M. Rangamani, S. F. Ross, D. T. Son and E. G. Thompson, "Conformal non-relativistic hydrodynamics from gravity," arXiv:0811.2049 [hep-th].

[10] M. Sakaguchi and K. Yoshida, "Super Schrodinger in Super Conformal," arXiv:0805.2661 [hep-th].

[11] M. Sakaguchi and K. Yoshida, "More super Schrodinger algebras from psu(2,2-4)," JHEP 0808, 049 (2008) arXiv:0806.3612 [hep-th]].

[12] D. Minic and M. Pleimling, "Non-relativistic AdS/CFT and Aging/Gravity Duality," arXiv:0807.3665 [cond-mat.stat-mech].

[13] A. V. Galajinsky, "Remark on quantum mechanics with conformal Galilean symmetry," Phys. Rev. D 78, 087701 (2008) arXiv:0808.1553 [hep-th]].

[14] S. Kachru, X. Liu and M. Mulligan, "Gravity Duals of Lifshitz-like Fixed Points," Phys. Rev. D 78, 106005 (2008) [arXiv:0808.1725 [hep-th]].

[15] P. Kovtun and D. Nickel, "Black holes and non-relativistic quantum systems," arXiv:0809.2020 [hep-th]. 
[16] C. Duval, M. Hassaine and P. A. Horvathy, "The geometry of Schródinger symmetry in gravity background/non-relativistic CFT," arXiv:0809.3128 [hep-th].

[17] D. Yamada, "Thermodynamics of Black Holes in Schroedinger Space," arXiv:0809.4928 [hep-th].

[18] F. L. Lin and S. Y. Wu, "Non-relativistic Holography and Singular Black Hole," arXiv:0810.0227 [hep-th].

[19] S. A. Hartnoll and K. Yoshida, "Families of IIB duals for nonrelativistic CFTs," arXiv:0810.0298 [hep-th].

[20] M. Schvellinger, "Kerr-AdS black holes and non-relativistic conformal QM theories in diverse dimensions," JHEP 0812, 004 (2008) [arXiv:0810.3011 [hep-th]].

[21] L. Mazzucato, Y. Oz and S. Theisen, "Non-relativistic Branes," arXiv:0810.3673 [hepth].

[22] P. Horava, "Quantum Criticality and Yang-Mills Gauge Theory," arXiv:0811.2217 [hepth].

[23] Y. Nakayama, S. Ryu, M. Sakaguchi and K. Yoshida, "A family of super Schrodinger invariant Chern-Simons matter systems," JHEP 0901, 006 (2009) arXiv:0811.2461 [hep-th]].

[24] A. Akhavan, M. Alishahiha, A. Davody and A. Vahedi, "Non-relativistic CFT and Semi-classical Strings," arXiv:0811.3067 [hep-th].

[25] A. Adams, A. Maloney, A. Sinha and S. E. Vazquez, "1/N Effects in Non-Relativistic Gauge-Gravity Duality," arXiv:0812.0166 [hep-th].

[26] M. Taylor, "Non-relativistic holography," arXiv:0812.0530 [hep-th].

[27] U. H. Danielsson and L. Thorlacius, "Black holes in asymptotically Lifshitz spacetime," arXiv:0812.5088 [hep-th].

[28] S. Pal, "Anisotropic gravity solutions in AdS/CMT," arXiv:0901.0599 [hep-th].

[29] J. P. Gauntlett, S. Kim, O. Varela and D. Waldram, "Consistent supersymmetric Kaluza-Klein truncations with massive modes," arXiv:0901.0676 [hep-th].

[30] A. Donos and J. P. Gauntlett, "Supersymmetric solutions for non-relativistic holography," arXiv:0901.0818 [hep-th].

[31] M. Alishahiha and A. Ghodsi, "Non-relativistic D3-brane in the presence of higher derivative corrections," arXiv:0901.3431 [hep-th].

[32] A. Akhavan, M. Alishahiha, A. Davody and A. Vahedi, "Fermions in non-relativistic AdS/CFT correspondence," arXiv:0902.0276 [hep-th]. 
[33] A. Bagchi and R. Gopakumar, "Galilean Conformal Algebras and AdS/CFT," arXiv:0902.1385 [hep-th].

[34] C. A. Fuertes and S. Moroz, "Correlation functions in the non-relativistic AdS/CFT correspondence," arXiv:0903.1844 [hep-th].

[35] A. Volovich and C. Wen, "Correlation Functions in Non-Relativistic Holography," arXiv:0903.2455 [hep-th].

[36] Y. Nakayama, M. Sakaguchi and K. Yoshida, "Non-Relativistic M2-brane Gauge Theory and New Superconformal Algebra," arXiv:0902.2204 [hep-th].

[37] F. Correa, V. Jakubsky and M. S. Plyushchay, Annals Phys. 324, 1078 (2009) arXiv:0809.2854 [hep-th]].

[38] C. R. Hagen, "Scale and conformal transformations in galilean-covariant field theory," Phys. Rev. D 5, 377 (1972).

[39] U. Niederer, "The maximal kinematical invariance group of the free Schrodinger equation," Helv. Phys. Acta 45, 802 (1972).

[40] G. Burdet, J. Patera, M. Perrin and P. Winternitz, "The Optical Group And Its Subgroups," J. Math. Phys. 19, 1758 (1978).

[41] C. Duval, G. Burdet, H. P. Kunzle and M. Perrin, "Bargmann Structures And NewtonCartan Theory," Phys. Rev. D 31, 1841 (1985).

[42] C. Duval, G. W. Gibbons and P. Horvathy, "Celestial Mechanics, Conformal Structures, and Gravitational Waves," Phys. Rev. D 43, 3907 (1991) arXiv:hep-th/0512188].

[43] P. D. Alvarez, J. L. Cortes, P. A. Horvathy and M. S. Plyushchay, JHEP 0903, 034 (2009) arXiv:0901.1021 [hep-th]].

[44] M. Henkel and J. Unterberger, "Schroedinger invariance and space-time symmetries," Nucl. Phys. B 660, 407 (2003) arXiv:hep-th/0302187.

[45] C. Duval, M. Hassaine and P. A. Horvathy, "The geometry of Schródinger symmetry in gravity background/non-relativistic CFT,"arXiv:0809.3128 [hep-th].

[46] M. Alishahiha, R. Fareghbal, A. E. Mosaffa and S. Rouhani, "Asymptotic symmetry of geometries with Schrodinger isometry," arXiv:0902.3916 [hep-th].

[47] M. Henkel, "Schrodinger invariance in strongly anisotropic critical systems," J. Statist. Phys. 75, 1023 (1994) arXiv:hep-th/9310081|.

[48] P. D. Alvarez, J. Gomis, K. Kamimura and M. S. Plyushchay, Annals Phys. 322, 1556 (2007) arXiv:hep-th/0702014.

[49] J. M. Maldacena, "The large $\mathrm{N}$ limit of superconformal field theories and supergravity," Adv. Theor. Math. Phys. 2, 231 (1998) [Int. J. Theor. Phys. 38, 1113 (1999)] arXiv:hep-th/9711200. 
[50] J. D. Brown and M. Henneaux, "Central Charges in the Canonical Realization of Asymptotic Symmetries: An Example from Three-Dimensional Gravity," Commun. Math. Phys. 104, 207 (1986).

[51] E. Inonu and E. P. Wigner, "On the Contraction of groups and their represenations," Proc. Nat. Acad. Sci. 39, 510 (1953).

[52] W. Mueck and K. S. Viswanathan, "Conformal field theory correlators from classical scalar field theory on $A d S_{d+1}$," Phys. Rev. D 58, 041901 (1998) arXiv:hep-th/9804035.

[53] D. E. Berenstein, J. M. Maldacena and H. S. Nastase, "Strings in flat space and pp waves from $N=4$ super Yang Mills," JHEP 0204, 013 (2002) arXiv:hep-th/0202021|.

[54] Work in progress. 